\title{
Defining the Range of Cellular Components, Including Internal Antigens, That Can Serve as Targets of Graft Rejection
}

\author{
L.A. Lampson, M.A. Lampson and A.D. Dunne \\ Center for Neurologic Diseases, Department of Neurology, Brigham and Women's Hospital and Harvard \\ Medical School, Boston MA 02115, USA
}

The mechanisms underlying rejection of grafted neural tissue are still being defined. Mechanisms relevant to genetically engineered cells are of current interest. To date, attention has focused on major histocompatibility complex (MHC) antigens as targets of graft rejection. Yet even when there is no MHC disparity, as when the patient's own cells are genetically altered, there is still a potential for graft rejection, directed against the novel antigens. We illustrate this in a rat model.

\section{METHODS}

The 9L gliosarcoma line was made to express $E$. coli-derived b-galactosidase (b-gal). The b-gal is constitutively expressed as a cell-filling internal protein, revealed histochemically $/ 1 /$. In three experiments, syngeneic CDF hosts were immunized with b-gal before intracerebral implantation of b-gal + 9L cells. After three weeks, the rats were sacrificed, and sections through the brain were stained to reveal b-gal + cells. Controls included rats immunized with other proteins or unimmunized. For each brain, the slide showing the most tumor was selected for measurement of tumor area by image analysis.

\section{RESULTS}

In each experiment, immunization with b-gal significantly reduced growth of the b-gal + cells. For example, in one study the mean tumor areas for control and b-gal immunized groups were, respectively, 101 and 0.3 units $^{2} \quad(\mathrm{p}<0.05$, Wilcoxon "sum of ranks" test).

\section{DISCUSSION}

We have developed an experimental model that permits identification and quantitative analysis of individual transplanted cells, their dissemination in the brain, and their interactions with responding leukocytes $/ 1 /$. The model was used to illustrate that internal molecules can be targets of graft rejection.

Our findings are consistent with current understanding of $\mathrm{T}$ cell recognition. $\mathrm{T}$ cells recognize [antigen $+\mathrm{MHC}$ proteins], not free antigen. It is now appreciated that the [antigen $+\mathrm{MHC}$ ] complex is formed within the cell. In different contexts, molecules synthesized within the cell, or ingested molecules, may be the antigen source. This suggests two ways by which internal molecules could become targets of graft rejection (Table I).

Host $\mathrm{T}$ cells may recognize [foreign antigen + $\mathrm{MHC}$ ] on transplanted cells with matching $\mathrm{MHC}$ phenotypes. Alternatively, host macrophages may ingest foreign antigen, then present it, with the macrophage's own MHC, to host $\mathrm{T}$ cells.

TABLE 1

Internal molecules as target antigens

1. Direct attack of target cell. Antigen (AG) synthesized within target cell. [MHC+AG] formed internally, then expressed at surface.

2. Indirect attack. AG ingested by antigen-presenting cell (APC), then expressed at surface with APC's MHC. Adjacent target cell killed as bystander. 
The resultant immune/inflammatory cascade may lead to death of adjacent transplanted cells as "bystanders". This indirect mechanism is of particular interest where the transplanted cells may not themselves express MHC antigens (Lampson et al., this volume).

These findings draw attention to the potential of internal molecules to serve as targets for graft rejection, even when there is no MHC disparity. They are relevant when the patient's own cells have been genetically modified to express novel products.

\section{REFERENCE}

1. Lampson et al. Cancer Res 1992; 52: 1008-1025. 

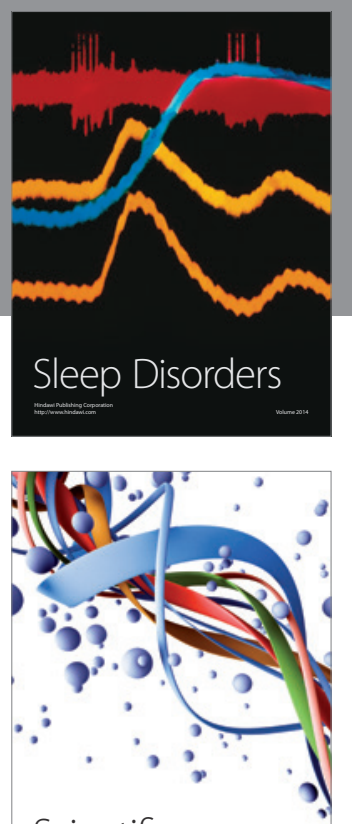

Scientifica
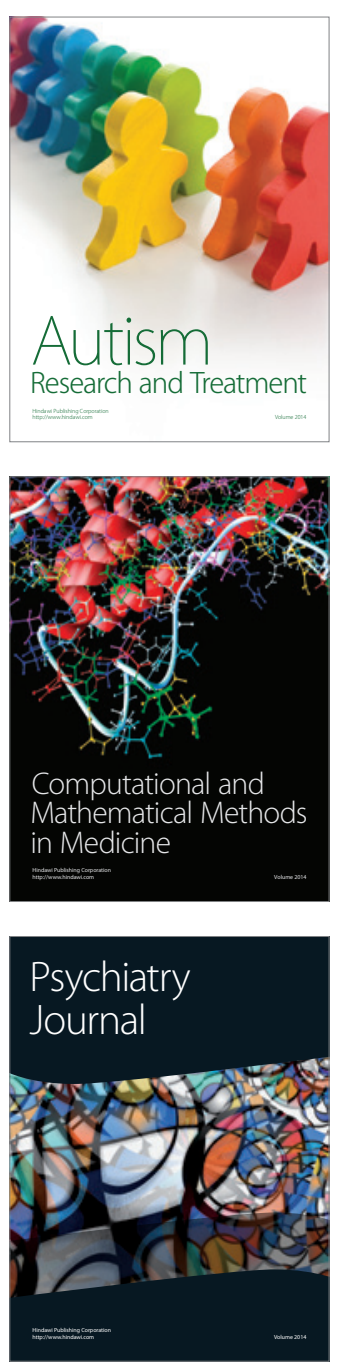
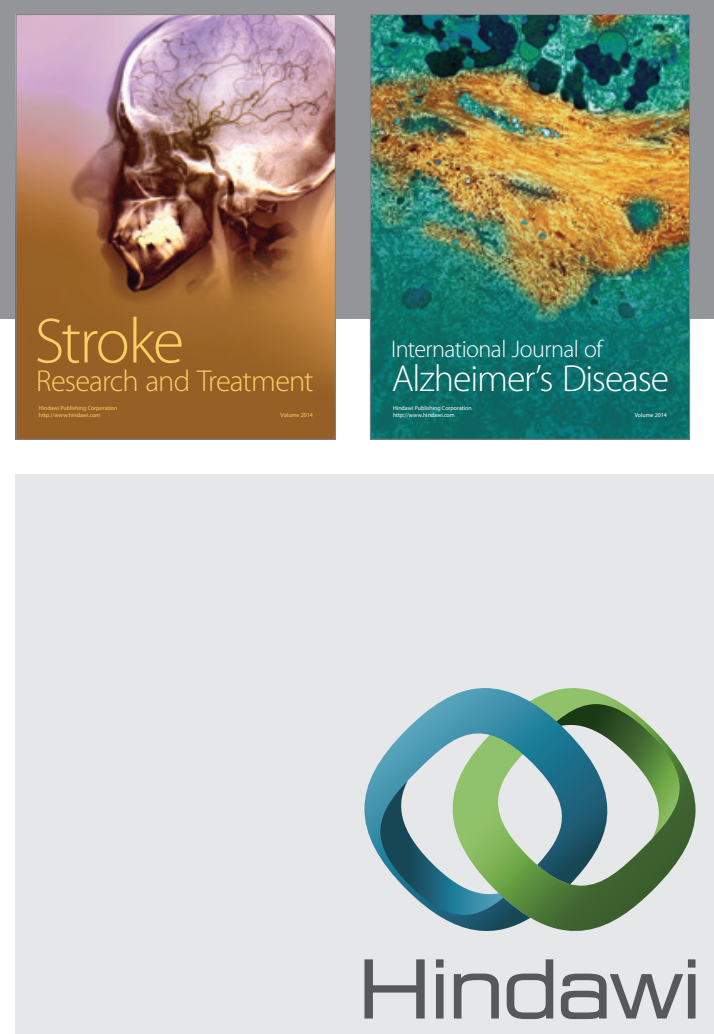

Submit your manuscripts at

http://www.hindawi.com
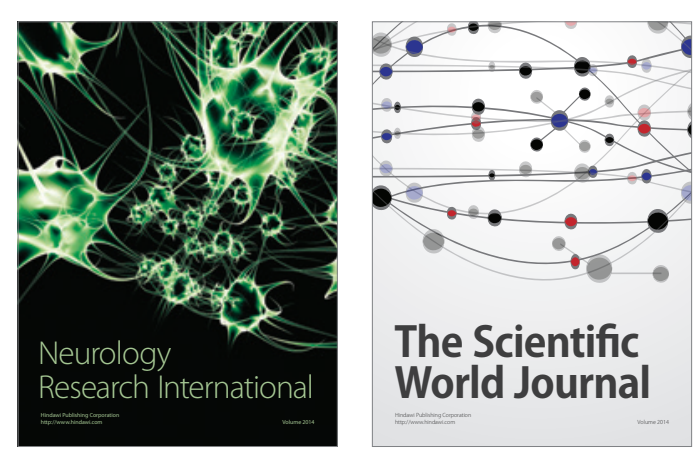

The Scientific World Journal

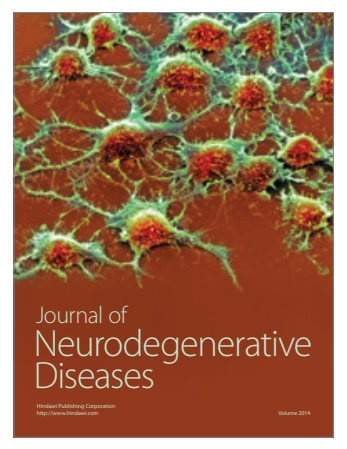

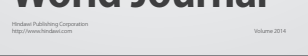

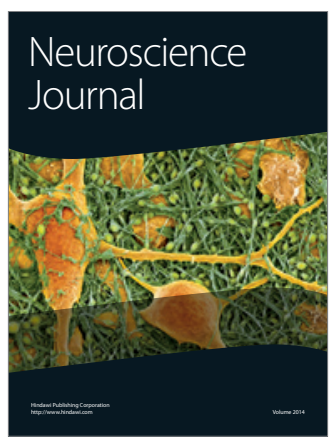

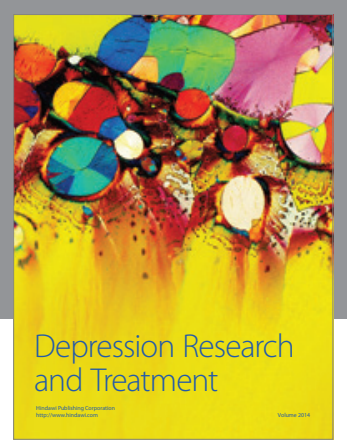
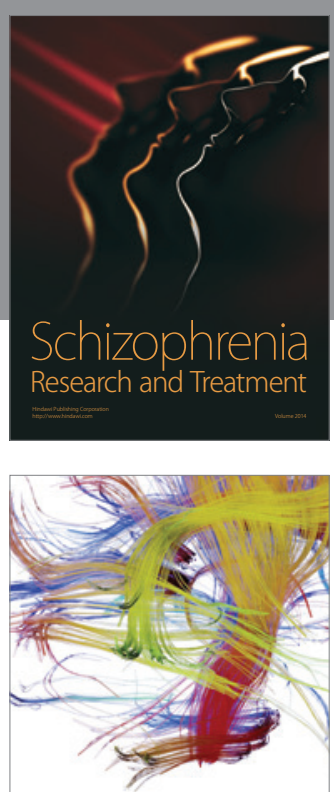

Brain Science

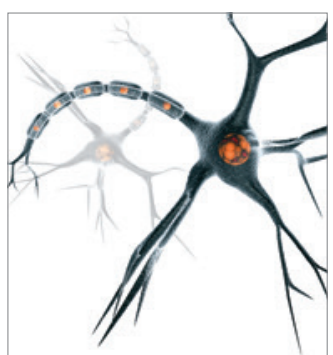

Neural Plasticity
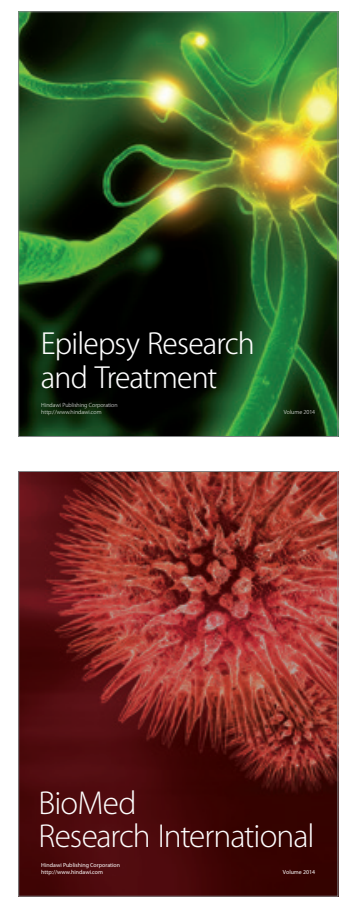

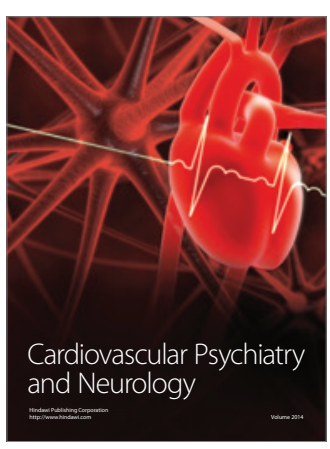

Parkinson's

Disease
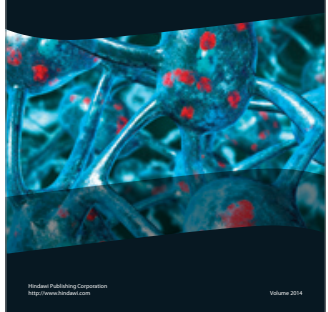